\title{
BMJ Open Variation in the recording of common health conditions in routine hospital data: study using linked survey and administrative data in New South Wales, Australia
}

\author{
Sanja Lujic, ${ }^{1}$ Diane E Watson, ${ }^{2}$ Deborah A Randall, ${ }^{1}$ Judy M Simpson, ${ }^{3}$ \\ Louisa R Jorm ${ }^{1}$
}

To cite: Lujic S, Watson DE, Randall DA, et al. Variation in the recording of common health conditions in routine hospital data: study using linked survey and administrative data in New South Wales, Australia. BMJ Open 2014;4:e005768. doi:10.1136/bmjopen-2014005768

- Prepublication history and additional material is available. To view please visit the journal (http://dx.doi.org/ 10.1136/bmjopen-2014005768).

Received 26 May 2014 Revised 14 July 2014 Accepted 4 August 2014

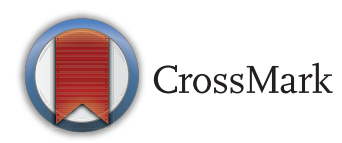

${ }^{1}$ Centre for Health Research, University of Western Sydney, Sydney, Australia

${ }^{2}$ National Health Performance Authority, Sydney, Australia ${ }^{3}$ School of Public Health, University of Sydney, Sydney, Australia

Correspondence to

Sanja Lujic;

s.lujic@uws.edu.au

\section{ABSTRACT}

Objectives: To investigate the nature and potential implications of under-reporting of morbidity information in administrative hospital data.

Setting and participants: Retrospective analysis of linked self-report and administrative hospital data for 32832 participants in the large-scale cohort study (45 and Up Study), who joined the study from 2006 to 2009 and who were admitted to 313 hospitals in New South Wales, Australia, for at least an overnight stay, up to a year prior to study entry.

Outcome measures: Agreement between self-report and recording of six morbidities in administrative hospital data, and between-hospital variation and predictors of positive agreement between the two data sources.

Results: Agreement between data sources was good for diabetes $(\kappa=0.79)$; moderate for smoking $(\kappa=0.59)$; fair for heart disease, stroke and hypertension ( $\kappa=0.40$, $\kappa=0.30$ and $\kappa=0.24$, respectively); and poor for obesity $(\kappa=0.09)$, indicating that a large number of individuals with self-reported morbidities did not have a corresponding diagnosis coded in their hospital records. Significant between-hospital variation was found (ranging from $8 \%$ of unexplained variation for diabetes to $22 \%$ for heart disease), with higher agreement in public and large hospitals, and hospitals with greater depth of coding.

Conclusions: The recording of six common health conditions in administrative hospital data is highly variable, and for some conditions, very poor. To support more valid performance comparisons, it is important to stratify or control for factors that predict the

completeness of recording, including hospital depth of coding and hospital type (public/private), and to increase efforts to standardise recording across hospitals. Studies using these conditions for risk adjustment should also be cautious of their use in smaller hospitals.

\section{INTRODUCTION}

Most nations with advanced economies publicly report on the comparative performance of hospitals with a view to accelerating and informing efforts to improve quality and allowing patients to make informed choices.

\section{Strengths and limitations of this study}

- The study was based on linked data from a large-scale cohort study and administrative hospital data to evaluate four health conditions and two health risk factors, as well as their combinations.

- The study used advanced multilevel modelling methods to comprehensively evaluate the recording of each morbidity in administrative data and quantify between-hospital variation.

- The study provides detailed information about how the validity of morbidity reporting varies among hospitals after accounting for patient factors.

- Limitations include the use of self-reported data in the absence of a 'gold standard' such as medical records.

Diagnoses recorded in administrative hospital data are commonly used in the construction and case-mix adjustment of hospital performance metrics, as well as for risk adjustment in epidemiological studies.

The construction of reliable health metrics relies on statistical methods that take into account the degree to which patients treated in different facilities have different morbidity and risk profiles that predispose them to requiring different interventions or to achieving different outcomes. These statistical methods, known as case-mix or risk adjustment, account for patient-related factors that are above and beyond the immediate control of healthcare professionals.

Thus, properly constructed performance metrics fairly reflect differences in healthcare experiences, patient outcomes and risks of adverse events. There has been some criticism of case-mix adjustments because they are subject to measurement error, ${ }^{1}$ but 
case-mix adjustment is still considered to be less biased than unadjusted comparisons. ${ }^{2}$

Most methods of case-mix adjustment rely principally on demographic and diagnostic information that is captured in administrative hospital data collections. The hospital data are collected and recorded in a database for administrative purposes, with clinical coders coding diagnostic information based on the patient's medical records. ${ }^{3}$ This approach may be suboptimal ${ }^{4}{ }^{5}$ because evidence from many countries suggests that administrative hospital data under-report the morbidity information needed to fully account for differences between hospitals in patient-related factors that predispose them to differences in measured outcomes. ${ }^{6-13}$ However, the impact of this under-reporting on comparative measures of hospital performance depends on whether it varies systematically among hospitals, because of differences in factors such as training or practice among coding staff, the comprehensiveness of clinicians' notes or 'upcoding' relating to funding models or incentives. ${ }^{14}$

This issue is relatively unexplored, aside from the work by Mohammed $e t a l^{2}$ which reported a non-constant relationship between case-mix variables and mortality among hospitals in the UK, explained by differences in clinical coding and admission practices across hospitals. These variations in coding accuracy were shown to be related to geographic location and bed size, with small rural facilities performing better than large urban hospitals. ${ }^{15}{ }^{16}$ In Australia, variations in the reporting and coding of secondary diagnoses in administrative hospital data have been shown to exist in public hospitals among Australian states, ${ }^{17}$ and also among hospitals within the state of New South Wales (NSW), with greater under-reporting in private and rural hospitals. ${ }^{3}$ However, the relative contributions of patient and hospital factors to these variations have not been identified, nor has this variation been formally quantified.

This study, using data linkage of survey and administrative data, aimed to further investigate the nature and potential implications of under-reporting of morbidity information in administrative hospital data by: (1) measuring the agreement between self-reported morbidity information and coded diagnoses; (2) quantifying the amount of between-hospital variation in this agreement and (3) identifying patient and hospital characteristics that predict higher or lower levels of agreement. We focused on clinical conditions common to case-mix and risk-adjustment models - diabetes, heart disease, hypertension and stroke. We also focus on smoking and obesity, due to their impact on health trajectories, rapid shifts in prevalence, substantial geographic variation in rates ${ }^{18}$ and paucity of international evidence on completeness of coding.

\section{METHODS}

Data sources

The 45 and Up Study

The 45 and Up Study is a large-scale cohort study involving 267153 men and women aged 45 years and over from the general population of NSW, Australia. The study is described in detail elsewhere. ${ }^{19}$ Briefly, participants in the 45 and Up Study were randomly sampled from the database of Australia's universal health insurance provider, Medicare Australia, which provides near-complete coverage of the population. People 80+ years of age and residents of rural and remote areas were oversampled. Participants joined the Study by completing a baseline questionnaire (available at https://www.saxinstitute.org.au/our-work/ 45-up-study/questionnaires/) between January 2006 and December 2009 and giving signed consent for follow-up and linkage of their information to routine health databases. About $18 \%$ of those invited participated and participants included about $11 \%$ of the NSW population aged 45 years and over. ${ }^{19}$

\section{The NSW Admitted Patient Data Collection}

The Admitted Patient Data Collection (APDC) includes records of all public and private hospital admissions ending in a separation, i.e. discharge, transfer, typechange or death. Each separation is referred to as an episode of care. Diagnoses are coded according to the Australian modification of the International Statistical Classification of Diseases and Health Related Problems 10th Revision (ICD-10-AM).$^{20} \mathrm{Up}$ to 55 diagnoses codes are recorded on the APDC, including the principal diagnosis and up to 54 additional diagnoses. Additional diagnoses are defined as "a condition or complaint either coexisting with the principal diagnosis or arising during the episode of care" in the Australian Coding Standards and should be interpreted as conditions that affect patient management. ${ }^{21}$ Assignment of diagnosis codes is done by trained clinical coders, using information from the patient's medical records.

The APDC from 1 July 2000 to 31 December 2010 was linked probabilistically to survey information from the 45 and Up Study by the NSW Centre for Health Record Linkage (http://www.cherel.org.au) using the 'best practice' protocol for preserving privacy. ${ }^{22}$

\section{Study population}

The study population comprised patients aged 45 years and above who participated in the 45 and Up Study and who had a hospitalisation lasting at least one night in the period up to 365 days prior to filling out the baseline 45 and Up Study survey. Day stay patients were excluded from the analysis to make the study more robust and generalisable beyond NSW and Australia, as there are differences in admission practices for same day patients between Australia and most other comparable countries. $^{23} \mathrm{NSW}$ is home to 7.4 million people, or one-third of the population of Australia.

\section{Measuring morbidity}

We examined four health conditions (diabetes, heart disease, hypertension and stroke) and two health risk factors (obesity and smoking), referred to hereafter collectively as 'morbidities'. For each participant, these 
health conditions were measured using self-report and administrative hospital data.

Self-reported morbidities were ascertained on the basis of responses to questions in the baseline 45 and Up Study survey. Diabetes, hypertension, stroke and heart disease were identified using the question "Has a doctor ever told you that you have [name of condition]?" Participants who did not answer the question were excluded from analyses $(n=1242)$.

Smoking was classified on the basis of answering "yes" to both of the questions "Have you ever been a regular smoker?" and "Are you a regular smoker now?" Participants' responses to the questions "How tall are you without shoes?" and "About how much do you weigh?" were used to derive body mass index (BMI), defined as body weight divided by height squared $\left(\mathrm{kg} / \mathrm{m}^{2}\right)$. The WHO's ${ }^{24}$ classification system was used to categorise individuals as obese $\left(\mathrm{BMI} \geq 30 \mathrm{~kg} / \mathrm{m}^{2}\right)$.

Morbidity information in administrative hospital data was ascertained using all 55 diagnosis codes in the APDC records (ICD-10-AM: E10-E14 for diabetes, I20I52 for heart disease, I60-I69, G45, G46 for stroke, I10I15 and R03.0 for hypertension, F17.2 or Z72.0 for smoking and E66 for obesity). The inclusion of broader ICD-10-AM codes for heart disease and stroke was chosen because of the broad definition of disease type in the self-reported data. Thus, heart disease codes were inclusive of coronary heart disease, pulmonary heart disease and other forms of heart diseases, including heart failure and arrhythmias. Stroke codes included cerebrovascular diseases without infarction among others.

\section{Predictors of agreement}

We explored patient-level as well as hospital-level factors as predictors of agreement between the two data sources.

Patient-level factors were self-reported in the 45 and Up Study baseline survey and included age, sex, education, country of birth, income and functional limitation. Functional limitation was measured using the Medical Outcomes Study-Physical Functioning scale, ${ }^{25}$ and classified into 5 groups: no limitation (score of 100), minor limitation (score 95-99), mild limitation (score 85-94), moderate limitation (60-84) and severe limitation (score 0-59).

Facility-level factors were type of hospital (public/ private), hospital peer group (akin to hospital size defined by number of case-mix weighted separations, ${ }^{26}$ which includes hospital remoteness in the classification), remoteness of hospital and depth of coding. Remoteness of the Statistical Local Area in which the hospital was located was classified according to the Accessibility/Remoteness Index of Australia (ARIA+), grouped into four categories (major city, inner regional, outer regional, remote/very remote). ${ }^{27}$ Depth of hospital coding was the mean number of additional diagnoses coded per episode of care for each hospital, calculated using all overnight hospitalisations for the full 45 and Up Study cohort from 2000 to 2010, and divided into four groups at the 25th, 50th and 75th centile. Hospital peer groups were divided into 5 categories: principal referral ( $\geq 25000$ separations per year), major (10 000-24 999 separations per year), district (20009999 separations per year), community (up to 2000 separations per year) and other (non-acute, unpeered hospitals). Missing information was treated as a separate category for any variables with missing data.

\section{Statistical methods}

We examined patient-level agreement between data sources for each of the six morbidities individually, as well as for their 15 two-way combinations. We compared the self-reported responses (yes/no) with all the diagnoses provided in the hospital records both for 'index' admissions and for the 'lookback' period admissions. ${ }^{28}$ The 'index' admission was the overnight hospital stay with admission date closest to the survey completion date and no longer than a year prior. Morbidity was coded as 'yes' if any of the diagnoses during that stay contained a mention of that morbidity. The 'lookback' admissions included all overnight stays in the 365-day period that preceded and included the 'index' admission. Morbidity was coded as 'yes' if any of the diagnoses from any lookback admissions contained a mention of that morbidity.

Agreement between the two data sources (yes/no) was measured using Cohen's $\kappa$ statistic. $\kappa$ Values above 0.75 denote excellent agreement, $0.40-0.75$ fair to good agreement and below 0.45 poor agreement. ${ }^{29}$ Agreement was computed for all 313 hospitals in the state, regardless of size, as well as for the 82 largest public hospitals, for which performance metrics are publicly reported.

Multilevel logistic regression was used to estimate OR with 95\% CIs for patient-level and hospital-level factors that predicted positive agreement between the two data sources. Multilevel models were chosen because of the clustering of patients within hospitals. Models were run for each of the six morbidities separately. These analyses were constrained to only those participants who selfreported the morbidity of interest, and the outcome was whether the index hospital record contained a mention of the morbidity or not. Addition of the hospital-level characteristics was done one at a time, due to the collinearity between variables. All ORs presented are adjusted for all other demographic variables in the model.

Variation at the hospital level was expressed as a median OR (MOR), which is the median of the ORs of pairwise comparisons of patients taken from randomly chosen hospitals, calculated as $\exp ^{0.954 \times \sqrt{\text { variance }} ;}, 30$ and the intraclass correlation coefficient (ICC), which is the percentage of the total variance attributable to the hospital level. ${ }^{31}$ Large ICCs indicate that differences among hospitals account for a considerable part of the variation in the outcome, whereas a small ICC means that the hospital effect on the overall variation is minimal. The 
relative influence of the hospital on reporting of morbidity was calculated using a variance partitioning coefficient expressed as a percentage of the total variance using the Snijders and Bosker latent variable approach. ${ }^{31}$

All data management was done using SAS V.9.2 $2^{32}$ and multilevel modelling using MLwiN V.2.24. ${ }^{33}$

The conduct of the 45 and Up Study was approved by the University of New South Wales Human Research Ethics Committee (HREC).

\section{RESULTS}

\section{Descriptive characteristics}

A total of 32832 study participants were admitted to 313 hospitals up to a year prior to completing the 45 and Up Study baseline survey. Just over half of the index admissions $(53 \%)$ were planned stays in hospital, and $57 \%$ were to a public hospital. Around one-third of the index admissions occurred within the 3 months before study entry, and the mean length of stay was 4.8 days (median $=3$ days). Just under half of the sample $(47 \%)$ reported having hypertension, with heart disease or obesity reported by $25 \%$ and current smoking by $6.1 \%$ of the sample. One-third (34\%) of participants had two or more morbidities (data not shown). Other characteristics of the sample at their index admission are shown in table 1. Characteristics of hospitals are summarised in table 2.

\section{Concordance between self-report and hospital records}

Overall, reporting of morbidity differed between the two data sources with 23257 (71\%) participants having at least one of the six self-reported morbidities, and 11977 $(36.5 \%)$ and $14335(43.7 \%)$ of the sample having at least one morbidity recorded on their index or lookback hospital admissions, respectively.

Table 3 gives the summary concordance measures for each morbidity and two-way morbidity combination. For the index admission, good agreement was found for diabetes $(\kappa=0.79)$; moderate agreement for smoking $(\kappa=0.59)$; fair agreement for heart disease $(\kappa=0.4)$; and poor agreement for stroke $(\kappa=0.3)$, hypertension $(\kappa=0.24)$ and obesity $(\kappa=0.09)$. In two-way combinations, moderate levels of agreement were found only for diabetes combinations (with smoking, hypertension and heart disease).

Incorporating a 1-year lookback period increased the numbers of participants with a morbidity recorded in a hospital record, with average relative increases in the $\mathrm{\kappa}$ values of 20\% (ranging from 2\% increase for smoking to $41 \%$ increase for obesity). Good to excellent level of agreements were still found only for diabetes $(\kappa=0.83)$ and smoking $(\kappa=0.6)$.

Agreement was only slightly higher among the 82 large public hospitals (see online supplementary table S1) with relative $\kappa$ values higher by $4 \%$, on average.
Table 1 Characteristics of the study sample at their index admission

\begin{tabular}{|c|c|c|}
\hline & \multicolumn{2}{|c|}{$\begin{array}{l}\text { All participants } \\
(\mathrm{N}=32832)\end{array}$} \\
\hline & $\overline{\mathbf{N}}$ & Per cent \\
\hline \multicolumn{3}{|l|}{ Demographic characteristics } \\
\hline \multicolumn{3}{|l|}{ Sex } \\
\hline Male & 16812 & 51.2 \\
\hline Female & 16020 & 48.8 \\
\hline \multicolumn{3}{|l|}{ Age } \\
\hline $45-59$ & 9666 & 29.4 \\
\hline $60-79$ & 16624 & 50.6 \\
\hline $80+$ & 6540 & 19.9 \\
\hline \multicolumn{3}{|l|}{ Country of birth } \\
\hline Australia & 25001 & 76.2 \\
\hline Other & 7448 & 22.7 \\
\hline Unknown & 383 & 1.2 \\
\hline \multicolumn{3}{|l|}{ Highest education level } \\
\hline No school & 5196 & 15.8 \\
\hline Year 10 or equivalent & 7894 & 24.0 \\
\hline Year 12 or equivalent & 2975 & 9.1 \\
\hline Trade & 4270 & 13.0 \\
\hline Certificate & 6109 & 18.6 \\
\hline University degree & 5662 & 17.3 \\
\hline Unknown & 726 & 2.2 \\
\hline \multicolumn{3}{|c|}{ Household income (\$, per annum) } \\
\hline$<20000$ & 9077 & 27.7 \\
\hline $20000-<50000$ & 8223 & 25.1 \\
\hline $500000-<70000$ & 2560 & 7.8 \\
\hline $70000+$ & 5042 & 15.4 \\
\hline Not disclosed & 6003 & 18.3 \\
\hline Missing & 1927 & 5.9 \\
\hline \multicolumn{3}{|l|}{ Functional status } \\
\hline No limitation & 4915 & 15.0 \\
\hline Mild limitation & 6011 & 18.3 \\
\hline Moderate limitation & 8701 & 26.5 \\
\hline Severe limitation & 10121 & 30.8 \\
\hline Missing & 3084 & 9.4 \\
\hline \multicolumn{3}{|l|}{ Admission characteristics } \\
\hline \multicolumn{3}{|l|}{ Admission type } \\
\hline Surgical & 15464 & 47.1 \\
\hline Other & 1439 & 4.4 \\
\hline Medical & 15929 & 48.5 \\
\hline \multicolumn{3}{|l|}{ Emergency status } \\
\hline Emergency & 13484 & 41.1 \\
\hline Planned & 17544 & 53.4 \\
\hline Other & 1803 & 5.5 \\
\hline \multicolumn{3}{|l|}{ Hospital of admission } \\
\hline \multicolumn{3}{|l|}{ Hospital type } \\
\hline Public & 18734 & 57.1 \\
\hline Private & 14096 & 42.9 \\
\hline \multicolumn{3}{|l|}{ Hospital remoteness } \\
\hline Major city & 19754 & 60.2 \\
\hline Inner regional & 8424 & 25.7 \\
\hline Outer regional & 4137 & 12.6 \\
\hline Remote/very remote & 363 & 1.1 \\
\hline \multicolumn{3}{|l|}{ Hospital depth of coding } \\
\hline 1-least comprehensive & 1629 & 5.0 \\
\hline 2 & 8803 & 26.8 \\
\hline 3 & 11543 & 35.2 \\
\hline 4-most comprehensive & 10857 & 33.1 \\
\hline \multicolumn{3}{|l|}{ Hospital peer group } \\
\hline Principal referral & 6329 & 19.3 \\
\hline Major & 11052 & 33.7 \\
\hline District & 6862 & 20.8 \\
\hline Community & 7018 & 21.4 \\
\hline Other & 1571 & 4.8 \\
\hline
\end{tabular}


Table 2 Characteristics of the hospital of admission

\begin{tabular}{lrr}
\hline & \multicolumn{2}{c}{ All hospitals $(\mathbf{N}=\mathbf{3 1 3})$} \\
\cline { 2 - 3 } & $\mathbf{N}$ & Per cent \\
\hline Hospital type & & \\
Public & 224 & 71.6 \\
Private & 88 & 28.1 \\
Hospital remoteness & & \\
Major city & 124 & 39.6 \\
Inner regional & 72 & 23.0 \\
Outer regional & 94 & 30.0 \\
Remote/very remote & 20 & 6.4 \\
Hospital depth of coding & & \\
1-least comprehensive & 48 & 15.3 \\
2 & 91 & 29.1 \\
3 & 89 & 28.4 \\
4-most comprehensive & 85 & 27.2 \\
Hospital peer group & & \\
Principal referral & 14 & 4.5 \\
Major & 33 & 10.5 \\
District & 51 & 16.3 \\
Community & 121 & 38.7 \\
Other & 94 & 30.0 \\
\hline
\end{tabular}

\section{Patient-level and hospital-level predictors of positive agreement}

The patient factors, which predicted positive agreement between the two data sources, differed between morbidities (table 4). Male sex was associated with better agreement for diabetes $(\mathrm{OR}=1.37,95 \%$ CI 1.19 to 1.58$)$, heart disease ( $\mathrm{OR}=1.30,95 \%$ CI 1.17 to 1.44$)$ and hypertension $(\mathrm{OR}=1.28,95 \%$ CI 1.18 to 1.38 ; see online supplementary table $\mathrm{S} 2$ ).

Older patients were significantly less likely to have smoking (80+years $\mathrm{OR}=0.48,95 \%$ CI 0.31 to 0.74 ) and obesity ( $\mathrm{OR}=0.14,95 \%$ CI 0.08 to 0.26 ) recorded in their hospital records and significantly more likely to have hypertension recorded ( $\mathrm{OR}=1.32,95 \%$ CI 1.16 to 1.49 ), compared with younger patients (45-59 years). People with higher levels of functional limitation were significantly more likely to have hypertension, diabetes and obesity recorded on their most recent hospital stay. Planned admissions to hospital had lower odds of having any of the six conditions recorded, as did medical admissions (for diabetes, smoking and obesity only). Agreement did not vary significantly for any other patient factors.

The four hospital-level covariates (hospital type, hospital peer group, hospital remoteness and depth of coding) were added to multilevel models (including a random intercept for hospital) one at a time, separately. Positive agreement between self-report and hospital records was significantly lower for hospitals with lower depth of coding across all morbidities. The odds of recording were also lower among private hospitals for all six morbidities, with this difference being statistically significant for hypertension, heart disease and stroke only. Records from smaller hospitals (district and community peer groups) were significantly less likely to agree with self-reported data on hypertension, diabetes and heart disease. Positive agreement did not vary significantly with remoteness of hospital, with the exceptions of diabetes (lower agreement for outer regional, remote and very remote hospitals) and smoking (lower agreement for remote and very remote hospitals; see online supplementary table S3).

\section{Quantifying variation between hospitals}

Before any hospital-level variables were added into the multilevel model, ICC indicated that between $8 \%$ (diabetes) and 22\% (heart disease) of the residual (unexplained) variation in agreement was attributable to the hospital after adjustment for the patient-level factors (table 5). This equated to MORs of 1.64 and 2.48, respectively, indicating that a patient in one hospital had an average of between $64 \%$ and $148 \%$ higher odds of having a particular morbidity recorded than a patient in a hospital with lower levels of recording. Less variation at the hospital level was found for the recording of diabetes, smoking and stroke, while more variation at the hospital level was found for the recording of hypertension, heart disease and obesity. When the analyses were restricted to 82 large public hospitals only, the between-hospital variation decreased to between 2\% (stroke) and 13\% (hypertension), or MOR of 1.24 and 1.94 (figure 1). This between-hospital variation was still significant for all morbidities except for stroke. Between-hospital variation was further reduced once lookback admissions were used to identify morbidities.

The addition of hospital-level variables to multilevel models, one at a time, separately, helped ascertain which factors explained the variation between hospitals (table 5). The addition of hospital-level factors contributed to explaining (i.e. decreasing) the residual variation for all conditions, except obesity. For the other morbidities, differences in the depth of coding explained from $16 \%$ (smoking) to $42 \%$ (hypertension) of residual variation between hospitals, while hospital type (public/private) explained from $0 \%$ (smoking) to 59\% (stroke), and hospital peer group explained from $10 \%$ (hypertension) to $27 \%$ (diabetes) residual variation between hospitals.

\section{DISCUSSION}

Our study found that the concordance of administrative hospital and self-reported data varied between the six morbidities examined, with agreement ranging from good for diabetes; moderate for smoking; through to fair for heart disease; and poor for hypertension, stroke and obesity. We demonstrated considerable betweenhospital variation in the recording of these common health conditions. Smaller, but still significant, betweenhospital variation was found when restricting the analyses to the largest public hospitals in the state.

Previous studies have validated information recorded in NSW administrative hospital data for demographic factors, ${ }^{34} 35$ and recording of perinatal conditions, ${ }^{36-39}$ 
Table 3 Agreement measures between self-report and hospital data, index and lookback admissions, all public and private hospitals in New South Wales, Australia $\underline{(n=313)}$

\begin{tabular}{|c|c|c|c|c|c|c|c|c|c|c|c|c|}
\hline \multirow[b]{3}{*}{ Morbidities* } & \multicolumn{6}{|c|}{ Index admission } & \multicolumn{6}{|c|}{ Lookback admissions } \\
\hline & \multicolumn{2}{|c|}{45 and Up yes } & \multicolumn{2}{|c|}{45 and Up no } & \multicolumn{2}{|l|}{ к } & \multicolumn{2}{|c|}{45 and Up yes } & \multicolumn{2}{|c|}{45 and Up no } & \multicolumn{2}{|l|}{$\kappa$} \\
\hline & $\begin{array}{l}\text { APDC } \\
\text { yes }\end{array}$ & $\begin{array}{l}\text { APDC } \\
\text { no }\end{array}$ & $\begin{array}{l}\text { APDC } \\
\text { yes }\end{array}$ & $\begin{array}{l}\text { APDC } \\
\text { no }\end{array}$ & $\begin{array}{l}\text { Per } \\
\text { cent }\end{array}$ & $95 \% \mathrm{Cl}$ & $\begin{array}{l}\text { APDC } \\
\text { yes }\end{array}$ & $\begin{array}{l}\text { APDC } \\
\text { no }\end{array}$ & $\begin{array}{l}\text { APDC } \\
\text { yes }\end{array}$ & $\begin{array}{l}\text { APDC } \\
\text { no }\end{array}$ & $\begin{array}{l}\text { Per } \\
\text { cent }\end{array}$ & $95 \% \mathrm{Cl}$ \\
\hline Hypertension & 4767 & 10512 & 1434 & 16119 & 24.0 & (22.9 to 25.0 ) & 6260 & 9019 & 2051 & 15502 & 30.2 & (29.1 to 31.2$)$ \\
\hline Heart disease & 3639 & 4668 & 1942 & 22583 & 40.3 & (39.0 to 41.5 ) & 4673 & 3634 & 2697 & 21828 & 47.0 & (45.8 to 48.2 ) \\
\hline Diabetes & 3560 & 1234 & 347 & 27691 & 79.1 & (78.1 to 80.1 ) & 3928 & 866 & 479 & 27559 & 83.0 & (82.1 to 83.9 ) \\
\hline Stroke & 541 & 1939 & 306 & 30046 & 29.8 & (27.0 to 32.6 ) & 776 & 1704 & 488 & 29864 & 38.3 & (35.8 to 40.8 ) \\
\hline Smoking & 1205 & 804 & 727 & 30096 & 58.7 & (56.7 to 60.7 ) & 1411 & 598 & 1076 & 29747 & 60.1 & (58.2 to 61.9 ) \\
\hline Obesity & 551 & 7611 & 114 & 24556 & 9.1 & (7.3 to 10.9$)$ & 810 & 7352 & 209 & 24461 & 12.8 & (11.1 to 14.6$)$ \\
\hline $\begin{array}{l}\text { Hypertension+heart } \\
\text { disease }\end{array}$ & 1172 & 3481 & 1270 & 26909 & 25.8 & (23.8 to 27.7 ) & 1807 & 2846 & 2008 & 26171 & 34.3 & (32.6 to 36.0 ) \\
\hline Hypertension+diabetes & 1819 & 1238 & 759 & 29016 & 61.3 & (59.6 to 62.9 ) & 2186 & 871 & 1021 & 28754 & 66.6 & (65.2 to 68.1$)$ \\
\hline Hypertension+stroke & 203 & 1317 & 189 & 31123 & 19.7 & (15.7 to 23.7 ) & 329 & 1191 & 340 & 30972 & 28.0 & (24.5 to 31.5 ) \\
\hline Hypertension+smoking & 133 & 598 & 180 & 31921 & 24.5 & (19.2 to 29.7 ) & 199 & 532 & 319 & 31782 & 30.6 & (26.0 to 35.2 ) \\
\hline Hypertension+obesity & 234 & 4574 & 93 & 27931 & 7.4 & (4.9 to 9.8$)$ & 383 & 4425 & 183 & 27841 & 11.5 & (9.2 to 13.9$)$ \\
\hline Heart disease+diabetes & 646 & 1154 & 404 & 30628 & 43.0 & (40.3 to 45.8 ) & 904 & 896 & 661 & 30371 & 51.2 & (48.9 to 53.6 ) \\
\hline Heart disease+stroke & 76 & 973 & 126 & 31657 & 11.2 & $(6.1$ to 16.4$)$ & 149 & 900 & 261 & 31522 & 19.0 & (14.4 to 23.5 ) \\
\hline Heart disease+smoking & 76 & 294 & 222 & 32240 & 22.0 & (15.3 to 28.6 ) & 118 & 252 & 373 & 32089 & 26.5 & (20.8 to 32.2 ) \\
\hline Heart disease+obesity & 79 & 1938 & 79 & 30736 & 6.4 & (2.5 to 10.4$)$ & 151 & 1866 & 169 & 30646 & 11.4 & (7.7 to 15.2$)$ \\
\hline Diabetes+stroke & 85 & 555 & 58 & 32134 & 21.1 & (15.0 to 27.3 ) & 140 & 500 & 119 & 32073 & 30.4 & (24.9 to 35.8 ) \\
\hline Diabetes+smoking & 143 & 161 & 108 & 32420 & 51.1 & (45.3 to 56.9 ) & 171 & 133 & 176 & 32352 & 52.1 & (46.7 to 57.4 ) \\
\hline Diabetes+obesity & 232 & 1701 & 65 & 30834 & 19.5 & (15.9 to 23.2 ) & 351 & 1582 & 120 & 30779 & 27.5 & (24.2 to 30.9 ) \\
\hline Stroke+smoking & 13 & 142 & 28 & 32649 & 13.1 & (0.1 to 26.1$)$ & 23 & 132 & 57 & 32620 & 19.3 & (7.8 to 30.8$)$ \\
\hline Stroke+obesity & 6 & 558 & 9 & 32259 & 2.0 & (0.0 to 10.0$)$ & 13 & 551 & 21 & 32247 & 4.2 & (0.0 to 11.9$)$ \\
\hline Smoking+obesity & 27 & 447 & 29 & 32329 & 9.9 & (1.9 to 17.9$)$ & 38 & 436 & 47 & 32311 & 13.2 & (5.5 to 20.9 ) \\
\hline
\end{tabular}




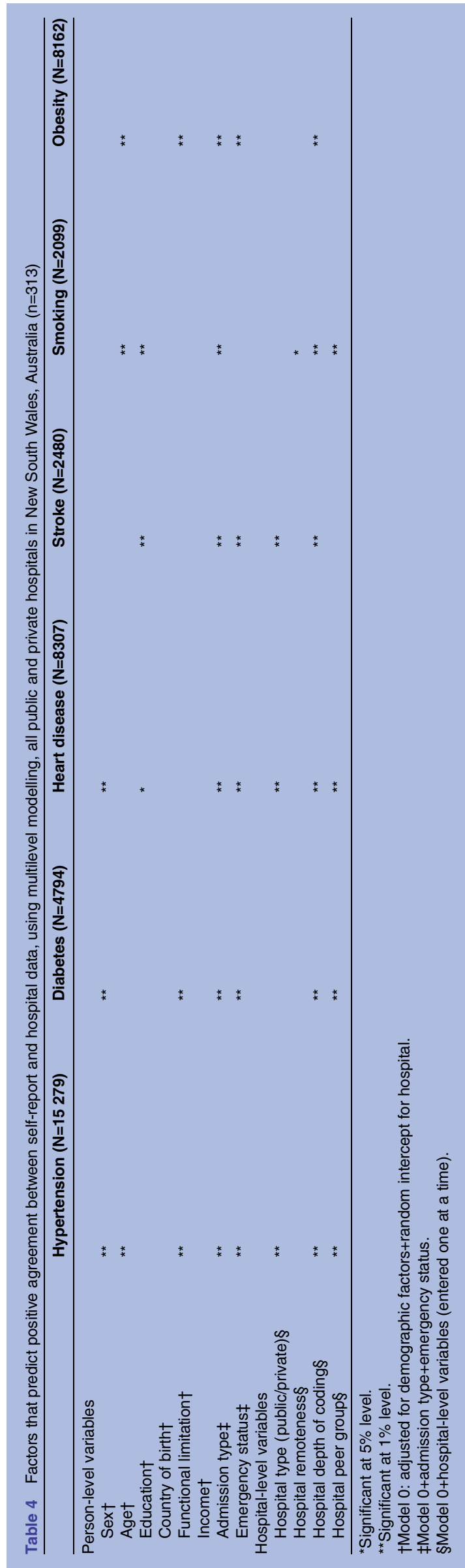

but there have been limited studies of the accuracy of the recording of health conditions commonly used for case-mix or risk adjustment. Our findings regarding agreement for the recording of diabetes $(\kappa=0.83)$ were similar to previous Australian studies, ${ }^{3}{ }^{10}$ while agreement for hypertension $(\kappa=0.3)$ and heart disease $(\kappa=0.47)$ was considerably lower in our study. These differences may be due to the fact that both previous studies used medical records as a 'gold standard', while we used self-report. Lower agreement rates for heart disease could be due to the broader range of heart disease types included in our study, with known lower levels of agreement for heart failure compared with myocardial infarction. ${ }^{9}{ }^{40}$ Higher sensitivities reported in a study from the state of Victoria ${ }^{10}$ could also be attributable to the differences in public hospital funding models between the two states. Specifically, Victoria has used activity-based funding since 1993, while this method of funding was introduced in NSW and other Australian states only subsequent to our study period. ${ }^{41}$ Introduction of activity-based funding has been shown to increase recording of additional diagnoses and procedures in Europe. ${ }^{42}$

Some of the apparent discrepancies in the levels of coding between conditions can be attributed to the coding rules that govern whether or not a diagnosis is recorded in administrative hospital data. Additional diagnoses, recorded on administrative hospital data, are coded only if they affect the patient's treatments received, investigations required and/or resources used during the hospital stay. Thus, diagnoses that relate to an earlier episode, and which have no bearing on the current hospital stay, are not coded for that particular stay. Therefore, it is not surprising that (managed) hypertension, in particular, might not be recorded in hospital data relating to, e.g. elective surgery. On the other hand, we found that diabetes is well recorded, suggesting that it is considered to affect patient management in most hospital stays, and possibly reflecting the impact of changes to the Australian Coding Standards for diabetes such that between 2008 and 2010, diabetes with complications could be coded even where there was no established cause and effect relationship between diabetes and the complication. ${ }^{43}$ It is for these reasons that researchers using administrative data sets are encouraged to incorporate information from previous hospitalisations, to increase the likelihood of capturing morbidity, as demonstrated in this as well as other Australian studies. ${ }^{44}$

As well as looking at single morbidities, ours is the first study, to our knowledge, to explore the variations of recording of multiple conditions in hospital data. Concordance of two-way condition combinations was very low, with best results found for combinations of diseases involving diabetes, which had the highest singlecondition level of agreement with self-reported data $(\kappa=0.83)$. Agreement measures for two-way combinations were found to be fair to good at best, with agreement on 
Table 5 Variance and ICC for hospital-level random effects from multilevel logistic regression, all public and private hospitals in New South Wales, Australia $(n=313)$

\begin{tabular}{|c|c|c|c|c|c|c|c|}
\hline & & $\begin{array}{l}\text { Hypertension } \\
\text { (N=15 279) }\end{array}$ & $\begin{array}{l}\text { Diabetes } \\
(\mathrm{N}=4794)\end{array}$ & $\begin{array}{l}\text { Heart } \\
\text { disease } \\
(\mathrm{N}=8307)\end{array}$ & $\begin{array}{l}\text { Stroke } \\
(\mathrm{N}=2480)\end{array}$ & $\begin{array}{l}\text { Smoking } \\
(\mathrm{N}=2099)\end{array}$ & $\begin{array}{l}\text { Obesity } \\
(\mathrm{N}=8162)\end{array}$ \\
\hline \multicolumn{8}{|c|}{ Hospital-level variance (SE)* } \\
\hline Model 0 & Patient factors & $0.80(0.10)$ & $0.27(0.06)$ & $0.91(0.12)$ & $0.38(0.10)$ & $0.35(0.09)$ & $0.68(0.14)$ \\
\hline Model 1 & $\begin{array}{l}\text { Model } 0 \\
\text { +hospital type } \\
\text { (public/private) }\end{array}$ & $0.65(0.08)$ & $0.27(0.06)$ & $0.71(0.10)$ & $0.16(0.06)$ & $0.35(0.09)$ & $0.69(0.14)$ \\
\hline Model 2 & $\begin{array}{l}\text { Model } 0 \\
\text { +hospital } \\
\text { remoteness }\end{array}$ & $0.77(0.09)$ & $0.25(0.05)$ & $0.92(0.12)$ & $0.37(0.10)$ & $0.33(0.08)$ & $0.68(0.14)$ \\
\hline Model 3 & $\begin{array}{l}\text { Model } 0 \\
\text { +hospital depth } \\
\text { of coding }\end{array}$ & $0.46(0.06)$ & $0.20(0.05)$ & $0.56(0.08)$ & $0.26(0.08)$ & $0.29(0.08)$ & $0.68(0.14)$ \\
\hline Model 4 & $\begin{array}{l}\text { Model } 0 \\
\text { +hospital peer } \\
\text { group }\end{array}$ & $0.72(0.09)$ & $0.21(0.05)$ & $0.75(0.10)$ & $0.34(0.09)$ & $0.31(0.08)$ & $0.67(0.14)$ \\
\hline$(\mathrm{ICC}(\%) \dagger$ & & 19.5 & 7.6 & 21.6 & 10.4 & 9.6 & 17.1 \\
\hline (MOR† & & 2.34 & 1.64 & 2.48 & 1.80 & 1.76 & 2.19 \\
\hline
\end{tabular}

three-way condition combinations (not investigated here) expected to be even lower. These findings have implications for research into multimorbidity (the co-occurrence of multiple chronic or acute diseases and medical conditions within one person ${ }^{45}$ ). We suggest that researchers who use administrative data for research into multimorbidity should use linked data to increase ascertainment and, if possible, supplement this information from other data sources, such as physician claims data or self-reported data.

We identified considerable between-hospital variability in the levels of recording of common health conditions, with between $8 \%$ and $22 \%$ of the variation attributable to hospital-level factors, after adjustment for patient factors. This was similar in magnitude to the variability previously reported for performance measures (varying from patient satisfaction, mortality, length of stay to quality of care) clustered at the facility level $(0-51 \%)^{46}$ and hospital-level variations in the use of services. ${ }^{47-49}$ Significant between-hospital variation was still present after constraining the analyses to the 82 largest public hospitals in the state.

The recording of hypertension and heart disease was particularly variable between hospitals, those with better reporting having on average 2.3 and 2.5 times, respectively, the odds of recording these conditions than those with lower levels of reporting. The corresponding figures were 1.9 and 1.6 times for the 82 largest hospitals in the state. These findings indicate the potential for reporting bias to influence comparisons of health performance indicators between hospitals, especially for indicators that use conditions such as heart disease or hypertension for case-mix adjustment. To our knowledge, no previous studies have provided detailed information about how
Figure 1 Variance for hospital-level random effects from multilevel logistic regression, for index and lookback admissions, by hospital size. *Significantly different from 0 at $5 \%$ level; ${ }^{* *}$ significantly different from 0 at $1 \%$ level.

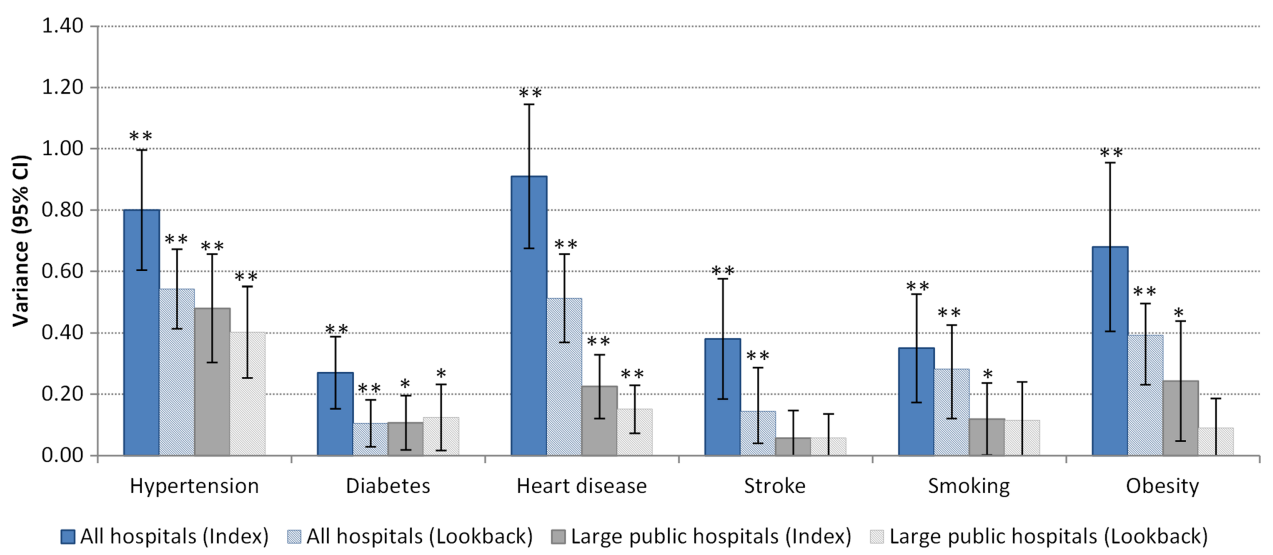


the validity of morbidity reporting varies among hospitals after accounting for patient factors.

Furthermore, we have shown that variations in the accuracy of morbidity reporting between hospitals are predominantly driven by the hospital's depth of coding -concordance between self-reported and hospital data is lower in hospitals with a lower average number of additional diagnoses recorded. Up to $42 \%$ of the variation in recording at the hospital level could be attributed to differences in hospital depth of coding. Even though the measure of depth of coding we used was crude, and related to hospital size, it still helps in highlighting the impact of coding practices on variations among hospitals. Other research using the same depth of coding measure has shown that the lower depth of coding can disproportionately disadvantage hospitals' standardised mortality ratios, one of the commonly reported measures of hospital performance. ${ }^{2}$ It will be important to track changes in the levels of the depth of coding across Australian states, and to consider the implications of these for state-based performance comparisons, following the national rollout of activity-based funding and comparative performance reporting.

Several factors might explain variation in depth of coding between hospitals. Clinical coders can code only information that has been recorded in the patient's medical record, so varying level of details recorded by clinicians will influence what gets coded. The training and professional development opportunities for coding staff might also influence the depth of coding. Also, case-mix funding systems, such as the Diagnosis Related Group (DRG) classification, are prone to 'upcoding' in order for services to receive higher reimbursement costs. ${ }^{14}$

We found that the reporting of conditions varied with hospital size, larger metropolitan hospitals having higher concordance, with $\kappa$ values higher by $7 \%$ on average when comparing large tertiary with smaller urban hospitals. This finding echoes those of Powell et $a l^{3}$ in NSW, Australia, during 1996-1998 and Rangachari ${ }^{16}$ in the USA, during 2000-2004. Our study showed that large tertiary hospitals had better concordance for the recording of hypertension and heart disease than smaller urban hospitals, but the reverse was true for stroke and smoking. Our finding that between-hospital variation in the recording of morbidities was up to two times higher when all hospitals, rather than just the largest ones, were included has implications for further research using data from smaller hospitals. This high variability in concordance among smaller hospitals may mean that morbidity-adjusted comparisons are not as valid as for larger hospitals. Researchers using information from these hospitals are encouraged to supplement their data with either self-report information and/or data linkage. The value-add of incorporating previous hospitalisations was also highlighted in our results for stroke and obesity, with 43-47\% more patients identified using lookback admissions than from a single admission only.
A particular strength of our study lies in the use of linked data from a large-scale cohort study to comprehensively evaluate the recording of common conditions in hospital data, and explore the variation in recording among hospitals. The 45 and Up Study contains records for one in every 10 persons aged 45 and over in NSW, so it provides a rich resource to answer research questions. Additionally, we used advanced multilevel modelling methods to quantify the amount of between-hospital variation in the level of recording of common health conditions, a finding which is of importance for research and policy paradigms due to its impact on adjusted comparisons among hospitals and the highlighted need to improve consistency of recording in hospitals across the state. To date, hospital-level variation has only been explored with a set outcome (e.g. mortality, readmission) in mind.

A potential limitation of our study was its use of selfreported information to explore concordance, in the absence of another 'gold standard', such as medical records. Access to medical records was not possible given the de-identified nature of our data and the large number of records in the data set. Moreover, studies that have examined accuracy of self-reported conditions against medical records have found high levels of agreement, ranging from $81 \%^{50}$ to $87 \%^{51}$ for hypertension, $66 \%^{40}$ to $96 \%^{50}{ }^{51}$ for diabetes and $60 \%{ }^{50}$ to $98 \%{ }^{52}$ for acute myocardial infarction. Validation studies in the 45 and Up Study cohort have reported strong correlations and excellent levels of agreement between self-reported and measured height and weight, and derived $\mathrm{BMI}^{53}$ as well as self-reported diabetes. ${ }^{54}$ Although the 45 and Up Study had a response rate of $18 \%$, the study sample is very large and has excellent heterogeneity. Furthermore, exposure-outcome relationships estimated from the 45 and Up Study data have been shown to be consistent with a large 'representative' population survey of the same population. ${ }^{55}$

\section{CONCLUSION}

The recording of common comorbid conditions in routine hospital data is highly variable and, for some conditions, very poor. Recording varies considerably among hospitals, presenting the potential to introduce bias into risk-adjusted comparisons of hospital performance, especially for indicators that use heart disease or hypertension for risk adjustment. Furthermore, betweenhospital variation is amplified when smaller and private hospitals are included in the analyses. Stratification of analyses according to factors that predict the completeness of recording, including hospital depth of coding and hospital type and size, supplementing morbidity information with linked data from previous hospitalisations and increases in efforts to standardise recording across hospitals, all offer potential for increasing the validity of risk-adjusted comparisons.

Acknowledgements The authors thank all of the men and women who participated in the 45 and Up Study. The 45 and Up Study is managed by the 
Sax Institute (http://www.saxinstitute.org.au) in collaboration with major partner Cancer Council NSW; and partners: the National Heart Foundation of Australia (NSW Division); NSW Ministry of Health; beyondblue; Ageing, Disability and Home Care, Department of Family and Community Services; the Australian Red Cross Blood Service; and UnitingCare Ageing. They would like to acknowledge the Sax Institute and the NSW Ministry of Health for allowing access to the data, and the Centre for Health Record Linkage for conducting the probabilistic linkage of records. They are grateful to $\mathrm{Dr}$ Fiona Blyth and $\mathrm{Dr}$ Kris Rogers for their advice at the early stages of the project.

Contributors SL had overall responsibility for the design of this study, data management, statistical analysis and drafting this paper. DEW and LRJ contributed to the conception and design of the study. LRJ helped with data acquisition and provided oversight for all analyses. DAR and JMS provided oversight and advice for the design and interpretation of the statistical analyses. All authors contributed to the interpretation of the findings, the writing of the paper and approved the final draft.

Funding The Assessing Preventable Hospitalisation InDicators (APHID) study is funded by a National Health and Medical Research Council Partnership Project Grant (\#1036858) and by partner agencies the Australian Commission on Safety and Quality in Health Care, the Agency for Clinical Innovation and the NSW Bureau of Health Information.

Competing interests None.

Ethics approval NSW Population and Health Services Research Ethics Committee and the University of Western Sydney Human Research Ethics Committee.

Provenance and peer review Not commissioned; externally peer reviewed.

Data sharing statement No additional data are available.

Open Access This is an Open Access article distributed in accordance with the Creative Commons Attribution Non Commercial (CC BY-NC 4.0) license, which permits others to distribute, remix, adapt, build upon this work noncommercially, and license their derivative works on different terms, provided the original work is properly cited and the use is non-commercial. See: http:// creativecommons.org/licenses/by-nc/4.0/

\section{REFERENCES}

1. Lilford R, Mohammed MA, Spiegelhalter D, et al. Use and misuse of process and outcome data in managing performance of acute medical care: avoiding institutional stigma. Lancet 2004:363:1147-54.

2. Mohammed MA, Deeks JJ, Girling A, et al. Evidence of methodological bias in hospital standardised mortality ratios: retrospective database study of English hospitals. BMJ 2009;338: b780.

3. Powell H, Lim LL, Heller RF. Accuracy of administrative data to assess comorbidity in patients with heart disease: an Australian perspective. J Clin Epidemiol 2001;54:687-93.

4. Pine M, Jordan HS, Elixhauser $\mathrm{A}$, et al. Enhancement of claims data to improve risk adjustment of hospital mortality. JAMA 2007;297:71-6.

5. Pine $\mathrm{M}$, Norusis $\mathrm{M}$, Jones $\mathrm{B}$, et al. Predictions of hospital mortality rates: a comparison of data sources. Ann Intern Med 1997; 126:347-54.

6. Hawker GA, Coyte PC, Wright JG, et al. Accuracy of administrative data for assessing outcomes after knee replacement surgery. $J$ Clin Epidemiol 1997:50:265-73.

7. Kieszak SM, Flanders WD, Kosinski AS, et al. A comparison of the Charlson comorbidity index derived from medical record data and administrative billing data. J Clin Epidemiol 1999;52:137-42.

8. Quan H, Parsons GA, Ghali WA. Validity of information on comorbidity derived rom ICD-9-CCM administrative data. Med Care 2002;40:675-85.

9. Preen DB, Holman CAJ, Lawrence DM, et al. Hospital chart review provided more accurate comorbidity information than data from a general practitioner survey or an administrative database. J Clin Epidemiol 2004;57:1295-304.

10. Henderson T, Shepheard J, Sundararajan V. Quality of diagnosis and procedure coding in ICD-10 administrative data. Med Care 2006;44:1011-19.

11. Quach S, Blais C, Quan H. Administrative data have high variation in validity for recording heart failure. Can J Cardiol 2010;26:e306-12.
12. Leal J, Laupland K. Validity of ascertainment of co-morbid illness using administrative databases: a systematic review. Clin Microbiol Infect 2010;16:715-21.

13. Chong WF, Ding YY, Heng BH. A comparison of comorbidities obtained from hospital administrative data and medical charts in older patients with pneumonia. BMC Health Serv Res 2011;11:105.

14. Steinbusch PJ, Oostenbrink JB, Zuurbier JJ, et al. The risk of upcoding in casemix systems: a comparative study. Health Policy 2007:81:289-99.

15. Lorence DP, Ibrahim IA. Benchmarking variation in coding accuracy across the United States. J Health Care Finance 2003;29:29.

16. Rangachari P. Coding for quality measurement: the relationship between hospital structural characteristics and coding accuracy from the perspective of quality measurement. Perspect Health Inf Manag 2007;4:3.

17. Coory M, Cornes S. Interstate comparisons of public hospital outputs using DRGs: are they fair? Aust N Z J Public Health 2005;29:143-8.

18. National Health Performance Authority. Healthy Communities: Overweight and obesity rates across Australia, 2011-12 (In Focus) 2013.

19. Banks E, Redman S, Jorm L, et al. Cohort profile: the 45 and up study. Int J Epidemiol 2008;37:941.

20. National Centre for Classification in Health. International Statistical Classification of Diseases and Related Health Problems, 10th Revision, Australian Modification (ICD-10-AM), Australian Classification of Health Interventions $(A C H I)$. Sydney: National Centre for Classification in Health, 2006.

21. Australian Institute of Health and Welfare. National health data dictionary. Version 16. Canberra: AlHW, 2012.

22. Kelman CW, Bass AJ, Holman CDJ. Research use of linked health data-a best practice protocol. Aust N Z J Public Health 2002;26:251-5.

23. Eagar K. Counting acute inpatient care. ABF Information Series No 5 2010. https://ahsri.uow.edu.au/content/groups/public/@web/@ chsd/documents/doc/uow082637.pdf (accessed Jul 2014).

24. World Health Organization. Obesity: preventing and managing the global epidemic. World Health Organization, 2000.

25. Stewart AL, Ware JE. Measuring functioning and well-being: the medical outcomes study approach. Duke University Press, 1992.

26. NSW Health. NSW Health Services Comparison Data Book 2008/ 09. 2010. http://www0.health.nsw.gov.au/pubs/2010/pdf/yellowbook 09.pdf (accessed Apr 2014).

27. Australian Institute of Health and Welfare. Regional and remote health: a guide to remoteness classifications. Canberra: AlHW, 2004.

28. Zhang JX, Iwashyna TJ, Christakis NA. The performance of different lookback periods and sources of information for Charlson comorbidity adjustment in Medicare claims. Med Care 1999;37:1128-39.

29. Fleiss JL, Levin B, Paik MC. The measurement of interrater agreement. Stat Methods Rates Proportions 1981;2:212-36.

30. Merlo J, Chaix B, Ohlsson $\mathrm{H}$, et al. A brief conceptual tutorial of multilevel analysis in social epidemiology: using measures of clustering in multilevel logistic regression to investigate contextual phenomena. J Epidemiol Community Health 2006;60:290-7.

31. Snijders T, Bosker R. Multilevel analysis. 2nd edn. London: Sage, 2012.

32. SAS Version 9.3 [software] [program]. Cary, North Carolina, 2010.

33. MLwiN Version 2.25 [software] [program]. Centre for Multilevel Modelling, University of Bristol, Bristol, 2012.

34. Bentley JP, Taylor LK, Brandt PG. Reporting of Aboriginal and Torres Strait Islander peoples on the NSW Admitted Patient Data Collection: the 2010 data quality survey. N S W Public Health Bull 2012;23:17-20.

35. Tran DT, Jorm L, Lujic S, et al. Country of birth recording in Australian hospital morbidity data: accuracy and predictors. Aust N Z J Public Health 2012;36:310-16.

36. Taylor LK, Travis S, Pym M, et al. How useful are hospital morbidity data for monitoring conditions occurring in the perinatal period? Aust N Z J Obstet Gynaecol 2005;45:36-41.

37. Roberts CL, Bell JC, Ford JB, et al. The accuracy of reporting of the hypertensive disorders of pregnancy in population health data. Hypertens Pregnancy 2008;27:285-97.

38. Hadfield RM, Lain SJ, Cameron CA, et al. The prevalence of maternal medical conditions during pregnancy and a validation of their reporting in hospital discharge data. Aust $N Z \mathrm{~J}$ Obstet Gynaecol 2008;48:78-82.

39. Lain SJ, Hadfield RM, Raynes-Greenow CH, et al. Quality of data in perinatal population health databases: a systematic review. Med Care 2012;50:e7-20 
40. Okura Y, Urban LH, Mahoney DW, et al. Agreement between self-report questionnaires and medical record data was substantial for diabetes, hypertension, myocardial infarction and stroke but not for heart failure. J Clin Epidemiol 2004:57:1096.

41. Eagar KM. What is activity-based funding? ABF Information Series No 1. 2011. http://ro.uow.edu.au/gsbpapers/47/ (accessed Apr 2014).

42. O'Reilly J, Busse R, Häkkinen U, et al. Paying for hospital care: the experience with implementing activity-based funding in five European countries. Health Econ Policy Law 2012;7:73.

43. Knight L, Halech R, Martin C, et al. Impact of changes in diabetes coding on Queensland hospital principal diagnosis morbidity data. 2011. http://www.health.qld.gov.au/hsu/tech_report/techreport_9.pdf (accessed Jul 2014)

44. Preen DB, Holman CDAJ, Spilsbury K, et al. Length of comorbidity lookback period affected regression model performance of administrative health data. J Clin Epidemiol 2006;59:940-6.

45. van den Akker M, Buntinx F, Knottnerus JA. Comorbidity or multimorbidity: what's in a name? A review of literature. Eur J Gen Pract 1996;2:65-70.

46. Fung V, Schmittdiel JA, Fireman B, et al. Meaningful variation in performance: a systematic literature review. Med Care 2010;48:140-8.

47. Bennett-Guerrero E, Zhao Y, O'Brien SM, et al. Variation in use of blood transfusion in coronary artery bypass graft surgery. JAMA 2010;304:1568-75.
48. Seymour CW, Iwashyna TJ, Ehlenbach WJ, et al. Hospital-level variation in the use of intensive care. Health Serv Res 2012;47:2060-80.

49. Randall DA, Jorm LR, Lujic S, et al. Disparities in revascularization rates after acute myocardial infarction between Aboriginal and non-Aboriginal people in Australia clinical perspective. Circulation 2013;127:811-19.

50. Merkin S, Cavanaugh K, Longenecker JC, et al. Agreement of self-reported comorbid conditions with medical and physician reports varied by disease among end-stage renal disease patients. J Clin Epidemiol 2007;60:634

51. Barber J, Muller S, Whitehurst T, et al. Measuring morbidity: self-report or health care records?. Fam Pract 2010;27:25-30.

52. Machón M, Arriola L, Larrañaga N, et al. Validity of self-reported prevalent cases of stroke and acute myocardial infarction in the Spanish cohort of the EPIC study. J Epidemiol Community Health 2013;67:71-5

53. $\mathrm{Ng} \mathrm{SP,} \mathrm{Korda} \mathrm{R,} \mathrm{Clements} \mathrm{M,} \mathrm{et} \mathrm{al.} \mathrm{Validity} \mathrm{of} \mathrm{self-reported} \mathrm{height}$ and weight and derived body mass index in middle-aged and elderly individuals in Australia. Aust N Z J Public Health 2011;35:557-63.

54. Comino EJ, Tran DT, Haas M, et al. Validating self-report of diabetes use by participants in the 45 and up study: a record linkage study. BMC Health Serv Res 2013;13:481.

55. Mealing NM, Banks E, Jorm LR, et al. Investigation of relative risk estimates from studies of the same population with contrasting response rates and designs. BMC Med Res Methodol 2010;10:26. 